\title{
Efficient smooth muscle cell differentiation of iPS cells on curcumin-incorporated chitosan/collagen/polyvinyl-alcohol nanofibers
}

\author{
Zakiye Mokhames $^{1} \cdot$ Zahra Rezaie $^{2} \cdot$ Abdolreza Ardeshirylajimi $^{2} \cdot$ Abbas Basiri $^{3} \cdot$ Mohammad Taheri $^{2} \cdot$ \\ Mir Davood Omrani ${ }^{3}$
}

Received: 28 December 2019 / Accepted: 16 March 2020 / Published online: 19 April 2020 / Editor: Tetsuji Okamoto

(C) The Society for In Vitro Biology 2020

\begin{abstract}
Bladder dysfunction is one of the most common diseases that occur for a number of reasons and the current treatment modalities do not improve much in its recovery process. Tissue engineering in the last two decades has given great hope for the treatment of these disorders. In this study, a composite nanofibrous scaffold was fabricated from chitosan, collagen, and polyvinyl-alcohol polymer blend while curcumin incorporated in scaffold fibers. The scaffold supportive functions from smooth muscle cell differentiation were studied when human-induced pluripotent stem cells were cultured on the scaffolds under differentiation medium. Biocompatibility of the fabricated scaffold increased significantly by incorporating curcumin in the scaffold fibers, where protein adsorption, cell attachment, and viability were increased in the nanofiber/curcumin group compared with the other groups. In addition, the expression level of smooth muscle cell-related genes, including alpha-smooth muscle actin $(\alpha \operatorname{SMA})$, smooth muscle 22 alpha (SM-22a), Caldesmon1, and Calponin1in the stem cells upregulated while cultured in the presence of curcumin, but this increase was significantly improved while cells cultured on the nanofibers/curcumin. In addition, $\alpha$ SMA protein in the cells cultured on the nanofibers/curcumin expressed significantly higher than those cells cultured on the nanofibers without curcumin. It can be concluded that smooth muscle cell differentiation of the induced pluripotent stem cells promoted by curcumin and this promotion was synergistically improved while curcumin incorporated in the nanofibers.
\end{abstract}

Keywords Curcumin $\cdot$ Nanofibers $\cdot$ Smooth muscle cell differentiation $\cdot$ Induced pluripotent stem cells $\cdot$ Tissue engineering

\section{Introduction}

Genitourinary disorders, traumas, cancers, inflammations, infections, injury due to treatment complications, or other conditions in the genitourinary tract can lead to complete organ damage or disability (Horst et al. 2019). Currently, genitourinary remodeling may be performed using one's own nonurologic tissues such as digestive tract, skin, or different parts

Mohammad Taheri

mohammad.taheri@sbmu.ac.ir

1 Department of Molecular Diagnostic, Emam Ali Educational and Therapeutic Center, Alborz University of Medical Sciences, Karaj, Iran

2 Urogenital Stem Cell Research Center, Shahid Beheshti University of Medical Sciences, Tehran, Iran

3 Urology and Nephrology Research Center, Shahid Beheshti University of Medical Sciences, Tehran, Iran of the body; tissues or heterologous materials such as bovine collagen; and homologous tissues and/or synthetic materials (Atala 2008). All of these materials often lead to various postreconstruction complications resulting from the rejection of transplanted tissue or potentially different performance parameters that cause system failure. This state of noncompliance can lead to metabolic complications, in addition to infection and other issues (Atala 2007). Therefore, definitely replacing lost or damaged urologic tissue with healthy tissues by reconstructive surgery can improve the genitourinary system. This can be achieved using tissue engineering and stem cell techniques (Selim et al. 2011; Orabi et al. 2013).

In bladder tissue engineering, scaffolds have been synthesized with the appropriate batteries and then stem cells will be cultured on the scaffolds and differentiate into the smooth muscle cells (SMC). Scaffold as a synthetic extracellular matrix (ECM) simulate the biological and mechanical activities of the natural ECM (Green and Elisseeff 2016). In fact, biomaterials can provide a three-dimensional (3D) and nanoscale space for the cells to transform into new tissues with 
proper functions and structure, as well as allowing the targeted delivery of the cells and bioactive factors (Ma 2008). Several natural and synthetic polymers have been introduced for use in tissue engineering and regenerative medicine (O'Brien 2011). Chitin and its acetylated compound are the most abundant natural polymers after cellulose. Chitosan has been studied numerously to produce the scaffolds for various tissue engineering applications in bone, skin, and cartilage. Because of its antibacterial, antiviral, non-toxic, and high tensile properties and also its biocompatibility and biodegradability (Islam et al. 2017), polyvinyl-alcohol (PVA) is the most abundant water-soluble synthetic polymer, produced from polymerization of vinyl acetate monomer to polyvinyl acetate and then hydrolysis to PVA. The polar nature and water solubility, non-toxicity, desirable adhesion properties, and high tensile strength make this polymer suitable for use in natural composites (Kadajji and Betageri 2011). It is one of the few polymers with simple carbon-carbon interconnections between the constituent units, which is perfect for biodegradation if suitable conditions are available (Marin et al. 2014). Collagen is the most abundant protein in the natural ECM of most tissues, and it is also widely used among natural polymers for use in polymeric scaffold construction. Collagen has a regular nanometer fiber structure with an overall 3D structure. The easy access to collagen and its unique structure led the researchers to pay special attention to this polymer (Dong and Lv 2016). Collagen is well adapted to the human biological system and its immune responses are quite similar to that of the host tissue. In addition, the collagen can be extracted from healthy tissues.

In addition to the scaffold, cells/stem cells and bio-factors are other two main parts of the tissue engineering construct that have critical roles in tissue regeneration. Bio-factors can be selected among growth factors, differentiation inducers, and other natural macromolecules. In this study, curcumin was selected as a bio-factor since its intrinsic toxicity is low and it has also numerous pharmacological activities such as antioxidant, anti-inflammatory, antimicrobial, and anticancer properties (Anand et al. 2007). Curcumin has also been traditionally known as a lipid lowering agent, liver protector, a cyclooxygenase inhibitor, protease inhibitor, free radical scavenger, and so on (Bengmark 2006).

The presence of cells in the designed scaffold is a major factor for tissue engineering success. Tissue regeneration requires a sufficient number of cells that do not stimulate the immune response. Different types of cells are used for tissue engineering applications, which can be classified into nonstem cells or adult cells and stem cells. Each of these cells has its advantages and disadvantages. Although mature cells are undeniably important for tissue engineering, the aggressive method of providing and low proliferative potential of these cells has led researchers to use stem cells as a promising alternative source of cells that target tissue (Caplan 2007).
Stem cells can be embryonic or adult, although embryonic stem cells (ESCs) are thought to be much more potent than adult stem cells (ASCs), there are several ethical and medicinal arguments for their use in humans. In addition, the invasive method of ASCs preparation, with age-dependent proliferative and differentiating features, has led to the introduction of induced pluripotent stem cells (iPSCs) for use in regenerative medicine in recent decades (Takahashi and Yamanaka 2006; Appasani and Appasani 2011). These cells are produced by reprogramming the patient's somatic cells and have proliferative and differentiating features such as embryonic stem cells, while lacking embryonic and adult stem cell problems.

In this project, a composite nanofibrous scaffold was fabricated by chitosan, PVA, collagen, and curcumin using electrospinning method. The potential of the scaffolds to support stem cell differentiation into bladder smooth muscle cells was evaluated after characterization of the scaffold and confirmation of its non-toxicity.

\section{Materials and Methods}

Composite nanofiber fabrication Chitosan (Mw: $550 \mathrm{kDa}$, Qingdao Ocean China) and collagen type-I (Col-I), which extracted from rat tail tendon, were dissolved in $90 \%$ acetic acid (Merck, Germany) to make a $2.5 \mathrm{wt} \%$ chitosan solution and a $5 \mathrm{wt} \%$ Col-I solution. PVA (98\% hydrolyzed, Mw: $72000 \mathrm{gmol}^{-1}$ ) was dissolved in heated $\left(80^{\circ} \mathrm{C}\right)$ deionized water to make a $5 \mathrm{wt} \%$ solution. The final solution was comprised from 30:20:50 ratio for chitosan, Col-I, and PVA, respectively. Curcuminincorporated nanofibers were fabricated by above solution containing about 5\% dispersed curcumin (Sigma, Adrich, St. Louis, MO). For electrospinning, solutions were poured out in two $5-\mathrm{ml}$ syringes and then placed $10 \mathrm{~cm}$ from an aluminum-covered collector with the flow rate of $0.6 \mathrm{ml} / \mathrm{h}$ while a $20 \mathrm{kV}$ voltage-supplier was applied.

Surface treatment Before scaffold characterization and cell seeding, scaffolds were plasma treated by a cylindrical quartz reactor $(2.45 \mathrm{GHz}$ frequency, Diener Electronics, Ebhausen, Germany) with oxygen gas supplier for introducing hydrophilicity to the surface of the scaffolds when placed in the chamber. Gas pressure was 0.4 mbar and glow discharge was $10 \mathrm{~min}$.

Wettability measurement To study hydrophilicity and wettability of the plasma-treated nanofibrous scaffolds, contact angle measurement was applied using the sessile drop method by a G10 Kruss contact angle goniometer. A drop of water was thrown on the scaffold surface and its contact angle was recorded after $10 \mathrm{~s}$. 
Scaffold morphological study Fabricated nanofibrous scaffolds were morphologically characterized using scanning electron microscopy (SEM) before and after cell seeding. Cell-cultured scaffolds were put in $2.5 \%$ glutaraldehyde for fixation and then put for $10 \mathrm{~min}$ in a graded series of ethanol (from 50 to $100^{\circ}$ ) for dehydration. Scaffolds were coated with gold and then imaged using a microscope (SEM, S-4500; Hitachi, Tokyo, Japan).

Curcumin release profile Curcumin release profile was studied according to the previously reported method (Golchin et al. 2019). In brief, curcumin-incorporated nanofibrous scaffolds were cut into certain dimensions and weight, then samples were incubated in phosphate buffered saline (PBS) while placed on the plate shaker at $37^{\circ} \mathrm{C}$. Every day, $500 \mu \mathrm{L}$ of PBS was removed and read by spectroscopy at $426 \mathrm{~nm}$ (Shimazu UV 1601); removed sample was also replaced with $500 \mu \mathrm{L}$ of fresh PBS. Curcumin concentration was calculated vs. optical density of a standard curve of curcumin ( 0.0 to $100 \mu \mathrm{g} / \mathrm{ml}$ ). Nanofibrous scaffold without curcumin was considered control.

Protein adsorption The rate of protein adsorption by fabricated nanofibrous scaffolds was studied based on the previously reported protocol (Saburi et al. 2019a); briefly, to do this, three solutions were prepared before doing experiment, including S-1: PBS (10 mM) and fetal bovine serum (FBS, $1 \% v / v)$, S-2: PBS and $2.0 \mathrm{wt} . \%$ sodium dodecylsulfate (SDS), and S-3: BSA calibration in SDS $(2.0 \mathrm{wt} \%)$ in PBS $(10 \mathrm{mM})$. After sterilization of the nanofibrous scaffold by UV irradiation (30 min) and alcohol (70\%, $1.5 \mathrm{~h}$ at RT), samples were cut into certain dimensions and placed in the S-1 for $1 \mathrm{~h}$ and then to release of attached proteins, scaffolds were placed in the S-2 for $20 \mathrm{~h}$. After that, protein concentrations of the S-1 and S-2 solutions were quantified using a protein assay kit (BCA; Pierce, Rockford, IL) at $530 \mathrm{~nm}$. The average protein uptake was reported in $\mu \mathrm{g}$ proteins per $\mathrm{mm}^{3}$ of samples calculated from S-3 solution.

Cell culture Frozen human iPSCs was prepared from the stem cell bank of department of tissue engineering and applied cell sciences (SBMU, Tehran, Iran). Frozen cells were thawed and cultured on the SNL76/7 cell line as a feeder while was inactivated by mitomycin-C under iPSCs culture medium according to the previously reported (Saburi et al. 2019b). For cell attachment and SMC differentiation studies, a cell density of $10^{5}$ cells $/ \mathrm{cm}^{2}$ and for viability assay a cell density of $2 \times 10^{4}$ cells $/ \mathrm{cm}^{2}$ were seeded on different groups such as the tissue culture polystyrene (TCPS), TCPS/curcumin, nanofibers, and nanofibers/curcumin. Cell attachment and viability assays were performed while iPSCs were cultured under basal medium (DMEM supplemented with 10\% FBS), and SMC differentiation procedure was performed while iPSCs were cultured
$21 \mathrm{~d}$ under SMC induction medium (DMEM containing about 5\% FBS, TGF $\beta 1$ ) (5 ng/ml, Peprotech, Rocky Hill, NJ) and Ascorbic Acid (AA, 30 mM, Merck, Darmstadt, Germany).

Viability assay Cell attachment and viability assays were carried out by MTT assay. To do MTT assay, $5 \mathrm{~h}$ (for cell attachment assay) and 1, 3, 5, and $7 \mathrm{~d}$ after seeding cells on the scaffolds and the cell culture plate as a control, MTT solution $(5 \mathrm{mg} / \mathrm{ml})$ was added to the cells. After 4-h cell incubation with a solution $\left(37^{\circ} \mathrm{C}\right.$ and $\left.5 \% \mathrm{CO}_{2}\right)$, medium was removed and DMSO was added as a solvent of Formazan crystals. Then optical density of the solutions was read at $570 \mathrm{~nm}$ using a microplate reader (BioTek Instruments, Inc., Winooski, VT) at $570 \mathrm{~nm}$.

Real-time RT-PCR To SMC-related gene expression evaluation, real-time RT-PCR was performed for alpha-smooth muscle actin (ASMA), smooth muscle 22 alpha (SM-22a), Caldesmon1, and Calponin1 in the SMC-differentiated cells on the scaffolds and cell culture plate as a control. First, total RNA of the samples was extracted by total RNA Prep kit (Biofact, Daejeon, South Korea) and then complementary DNA (cDNA) was synthesized by cDNA synthesis kit (Biofact, Daejeon, South Korea) according to the kit protocol. Then, real-time RT-PCR was carried out by prepared cDNAs for each sample $(5 \mu \mathrm{g} / \mathrm{ml})$, injectable water, primers (Table.1), and 2X real-time PCR master mix (SYBR Green, Biofact, Daejeon, South Korea) using the ABI StepOnePlus thermal cycler (Applied Biosystems, Foster City, CA).

Immunocytochemistry At the end of study, SMC differentiated on curcumin-incorporated nanofibrous scaffold and empty scaffold was fixed by paraformaldehyde (PFA, 4\%) for $30 \mathrm{~min}$ and then samples were placed in PBS supplemented with $0.4 \%$ Triton X-100 to make permeable $\left(10 \mathrm{~min}, 4^{\circ} \mathrm{C}\right)$. After that, blocking was performed using BSA $\left(1 \mathrm{~h}, 37^{\circ} \mathrm{C}\right)$ and then

Table 1. Primer sequences used in real-time RT-PCR analysis at this study

\begin{tabular}{cl}
\hline Gene & Primer sequences $\left(5^{\prime} \rightarrow 3^{\prime}\right)$ \\
\hline$\beta$-actin & GTCCTCTCCCAAGTCCACAC \\
& GGGAGACCAAAAGCCTTCAT \\
$\alpha$ SMA & GACGCACAACTGGCATC \\
& GCAGTAGTAACGAAGAATAGC \\
Calponin 1 & ATGTCCTCTGCTCACTTCAAC \\
& GCTGGTGGTCATACTTCTGG \\
SM22a & GVAGTVVAAAATGAGA \\
& CTGTTGCTGCCCATTTGAAG \\
Caldesmon1 & AGACTACAAGGTATCATTGG \\
& AAC \\
& GACAGGTCAGCAATCAGATG \\
\hline
\end{tabular}


anti-alpha smooth muscle actin (anti- $\alpha$ SMA) antibody (Catalog No. MAB1420) was added to the samples as primary antibody (overnight, $4^{\circ} \mathrm{C}$ ). Then, FITC-goat anti-rabbit IgG (Invitrogen, Carlsbad, CA) was also added as secondary antibodies to the samples following primary antibody removing. Finally, solutions were removed from the samples and then treated with DAPI ( $30 \mathrm{~s})$ before visualizing by an inverted fluorescent microscope.

Statistics Experiments were designed for three times repeating independently, all data reported as mean \pm standard deviation (SD). Using SPSS 17.0 software, simple one-way analysis of variance (ANOVA) was applied to compare data between the two groups and when the probability $(P)$ values were less than 0.05 and 0.01 , considered to be significant and showed by * and $* *$, respectively.

\section{Results}

Nanofiber characterizations SNL76/7 cell line was cultured on the cell culture plate and when its confluence reached to 80 90\% (Fig. 1A), cells were treated with mitomycin-C for $3 \mathrm{~h}$ and then human iPSCs were cultured on them. After $4 \mathrm{~d}$, iPSCs colonies were observed as a typical characteristic of the iPSCs (Fig. 1B). Fabricated chitosan/Col/PVA (nanofibers) (Fig. 1C) and curcumin-incorporated chitosan/Col/PVA (nanofibers/ curcumin) (Fig. 1D) scaffolds were morphologically characterized by SEM and obtained images demonstrated that scaffolds had fibers without bead and with nanometer diameters, interconnected pores, and random orientation. Wettability of the scaffolds was studied by contact angle test and results displayed that after plasma treatment nanofiber contact angle was decreased from 132 to $98^{\circ}$ while nanofiber/curcumin contact angle decreased from 109 to $72^{\circ}$.

The release of the curcumin from nanofibers was also evaluated every day during the period of study ( $21 \mathrm{~d}$ ) and results indicated that a burst release around $20 \%$ was occurring on the first day, but after that curcumin cumulative release was increased slowly until day 21 that almost $90 \%$ of the curcumin was released from the nanofibers (Fig. 2A). Then, the effects of curcumin on the scaffold's tendency to adsorb protein was evaluated and results revealed that the highest adsorption of the protein was detected in the nanofiber/curcumin group that was significantly higher than in the nanofiber, TCPS/curcumin, and TCPS groups; however, this property in nanofibers and TCPS/curcumin was almost similar but significantly higher than in the TCPS group (Fig. 2B).

Biocompatibility of the fabricated nanofibrous scaffolds was studied using cell attachment, SEM, and viability assays. The results obtained from iPSCs attachment assay were similar to the protein adsorption where the highest attachment was detected in the nanofiber/curcumin group that was significantly higher than in other groups (Fig. 2C). Although iPSCs attachment in the nanofiber and TCPS/curcumin groups was also significantly higher than its measure in the TCPS group. SEM results showed that iPSCs properly attached, proliferated, and expanded on the surfaces of both nanofibers (Fig. 3A)

Figure 1. Photographs of SNL76/7 cell line as a feeder layer before inactivating $(A)$ and human-induced pluripotent stem cells (iPSCs) cultured on the feeder layer a week after thawing (B); Photographs of chitosan/Col/ PVA $(C)$ and chitosan/Col/PVA/ curcumin $(D)$ nanofibrous scaffold obtained by SEM.
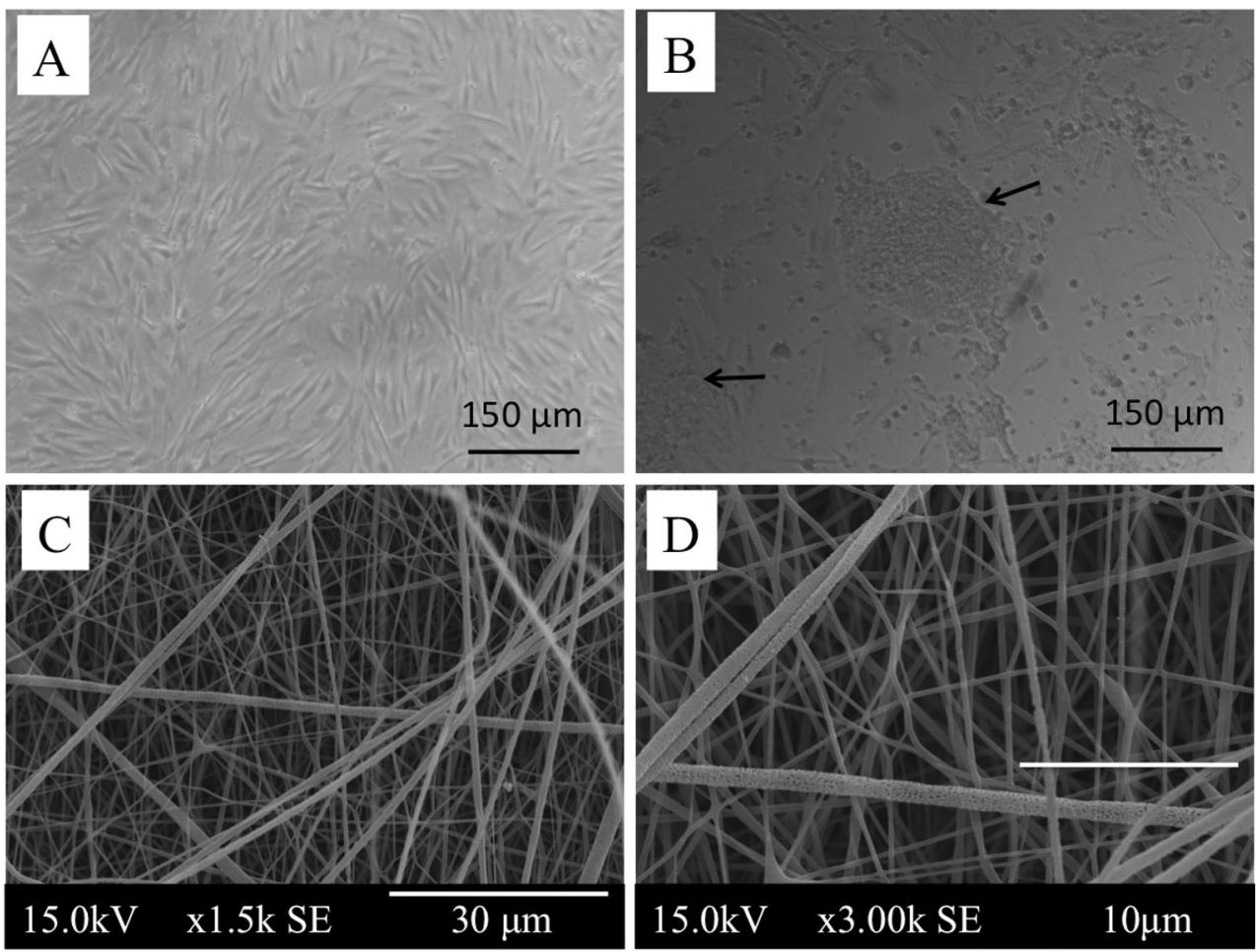
Figure 2. Cumulative curcumin release from chitosan/Col/PVA/ curcumin nanofibers $(A)$, protein adsorption $(B)$, and cell attachment $(C)$ results of tissue culture polystyrene (TCPS), TCPS/curcumin, chitosan/Col/ PVA (nanofibers), and chitosan/ $\mathrm{Col} / \mathrm{PVA} /$ curcumin (nanofibers/ curcumin) groups. The significant differences between groups are indicated with one star sign $(P<0.05)$ and two star sign $(P<0.01)$.
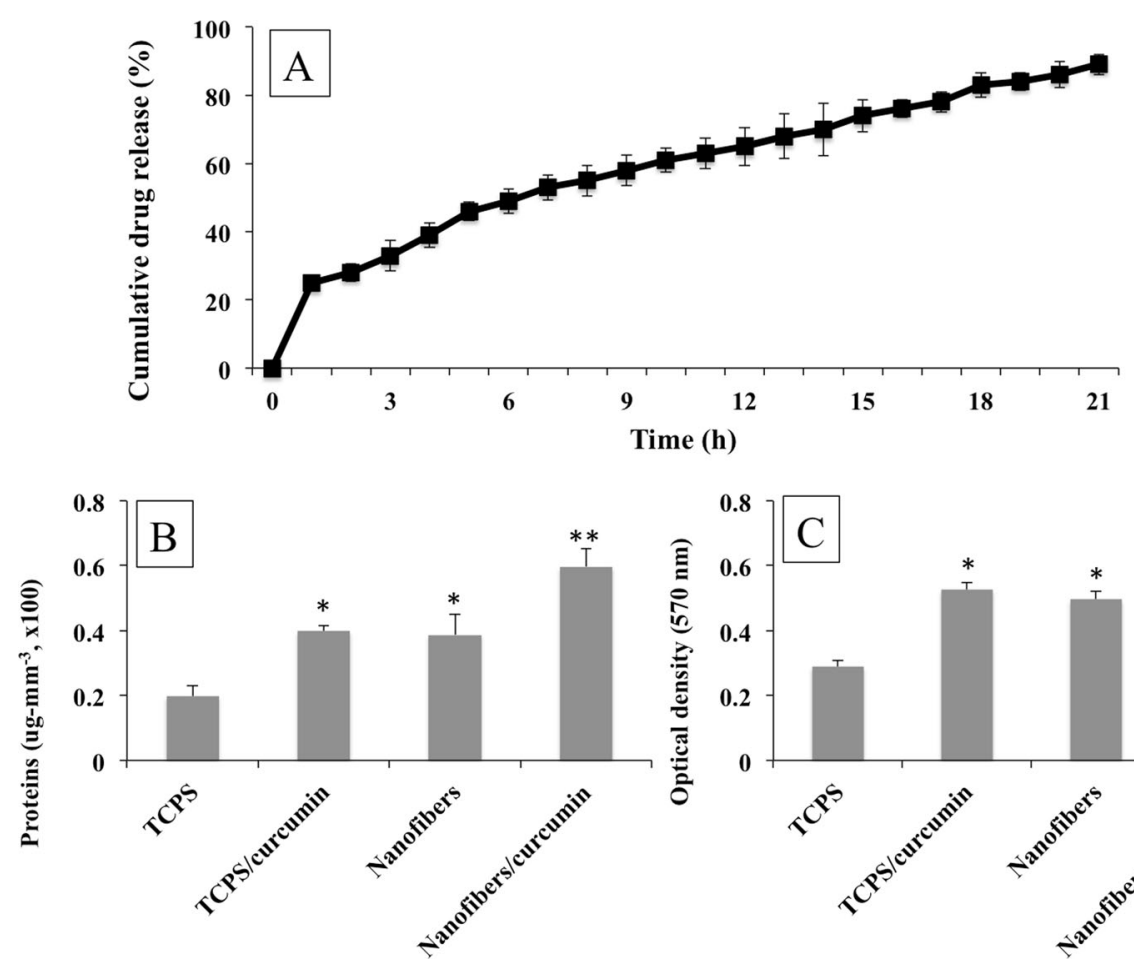

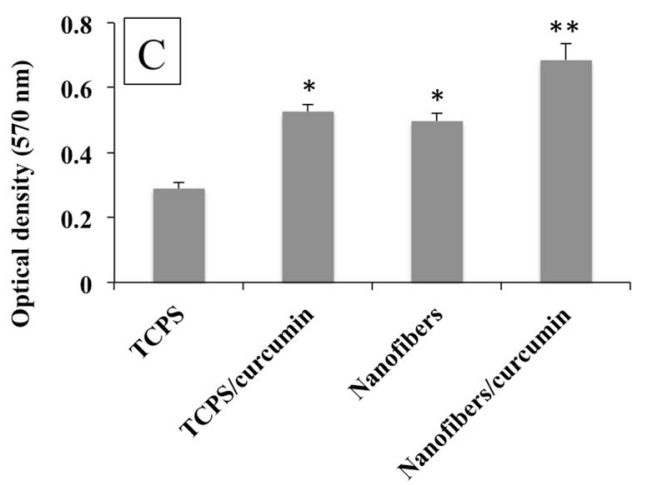

and nanofibers/curcumin (Fig. 3B). A quantitative biocompatibility test was carried out by evaluating the viability of the iPSCs when grown on the surface of nanofibers, nanofibers/ curcumin, TCPS/curcumin, and TCPS uses an MTT assay (Fig. 4). Obtained results revealed that the iPSCs growth and proliferation increased during the culture period in all groups. This increase in the nanofiber/curcumin group was significantly higher than in other groups at days 3,5 , and 7 . In addition, iPSCs growth and proliferation while cultured in the nanofiber group was also higher than those cells cultured on the TCPS/curcumin group, but was not significant, although this increase in both groups was significantly higher than those iPSCs cultured on the TCPS group at days 5 and 7 .

Differentiated cell characterization Four important SMCrelated gene markers such as $\alpha$ SMA, SM22-a, Calponin1, and Caldesmon 1 were studied during the period of study while human iPSCs grown and differentiated into SMC in the various groups including nanofibers, nanofibers/curcumin, TCPS/curcumin, and TCPS (Fig. 5). On all three time points (7, 14, and $21 \mathrm{~d}), \alpha \mathrm{SMA}$ was expressed in nanofiber/curcumin group significantly higher than in other groups, although this gene in nanofiber group was also expressed significantly higher than in the TCPS/curcumin and TCPS groups. In addition, this gene was also upregulated in the TCPS/curcumin group in comparison with TCPS group, but this difference was not significant. The highest expression level of the SM22-a was detected in the nanofiber/curcumin group on all $3 \mathrm{~d}$ compared with the other groups. The expression level of the SM22-a in nanofiber and TCPS/curcumin groups on days 7, 14, and 21 was also significantly upregulated compared with that in the TCPS group. Calponin1 and Caldesmon1 were expressed in nanofiber/curcumin group significantly higher than in the other two groups on all days of analysis. On day 7, expression levels
Figure 3. SEM photographs of human iPSCs-seeded chitosan/ Col/PVA $(A)$ and chitosan/Col/ PVA/curcumin $(B)$ nanofibrous scaffold obtained by SEM.
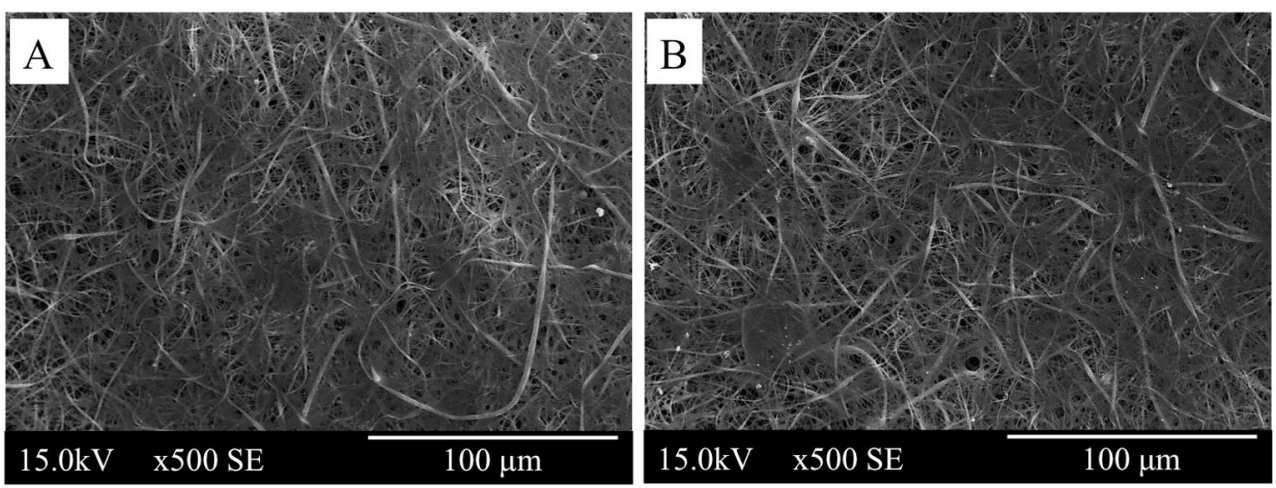
Figure 4. Viability of the human iPSCs cultured on tissue culture polystyrene (TCPS),

TCPS/curcumin, chitosan/Col/ PVA (nanofibers), and chitosan/ $\mathrm{Col} / \mathrm{PVA} /$ curcumin (nanofibers/ curcumin) groups during a wk culture period. The significant differences between groups are indicated with one star sign $(P<0.05)$.

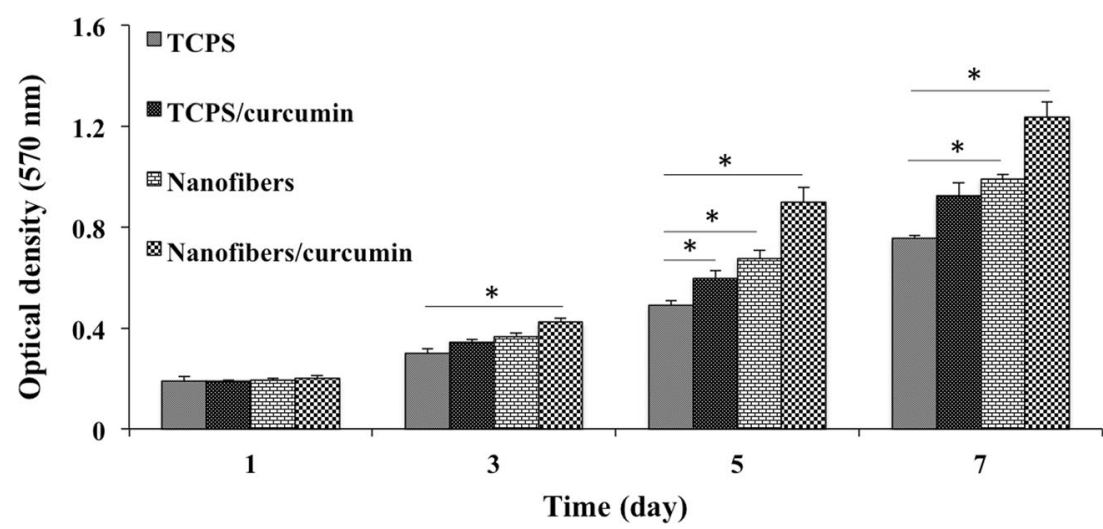

of these genes in the nanofiber, TCPS/curcumin, and TCPS groups were not significantly different, but on days 14 and 21 , their expression in the nanofiber group was significantly higher than in the TCPS/curcumin group and both groups (nanofibers, TCPS/curcumin) were also significantly higher than TCPS group.

For more detailed study of the fabricated nanofibrous scaffold's potential to support SMC differentiation process, $\alpha \mathrm{SMA}$ protein was stained using ICC in SMC-differentiated iPSCs grown on the nanofiber and nanofiber/curcumin groups. Quantification of the ICC images (Fig. 6E) demonstrated that $\alpha$ SMA protein expression level in the nanofiber/curcumin group (Fig. 6D) was significantly higher than in the nanofiber group (Fig. 6B).

\section{Discussion}

Tissue injury and organ loss caused by degenerative diseases or neoplasia is still a major challenge. Appropriate methods to replace damaged tissues require a variety of techniques that face many difficulties. Generally, end-stage limb failure is treated

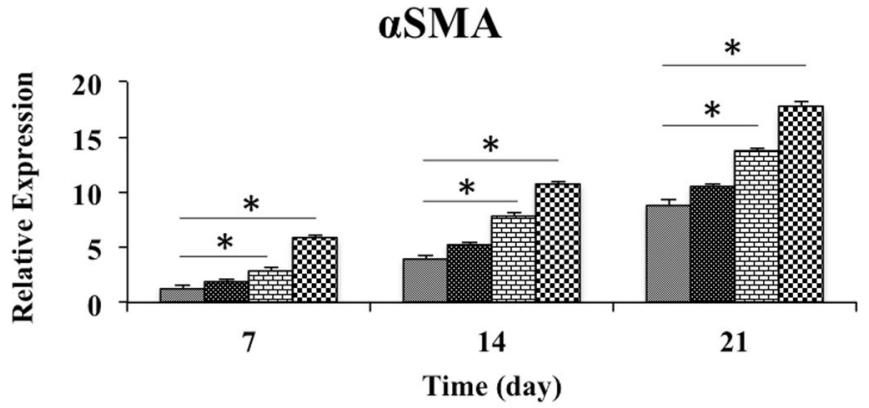

$\square$ TCPS

\section{Calponin1}

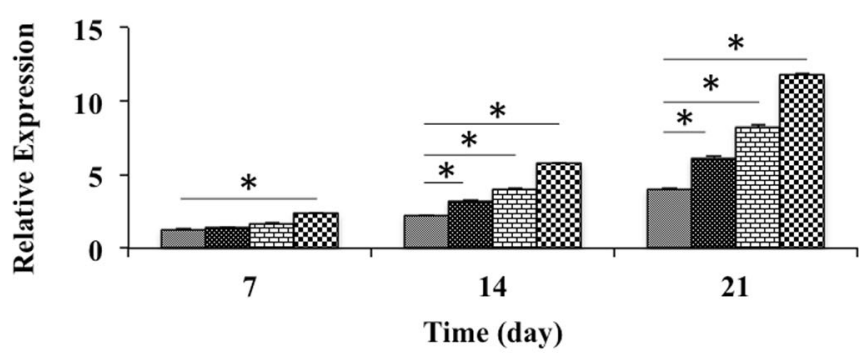

$\square$ TCPS TCPS/curcumin Nanofibers Nanofibers/curcumin

Figure 5. Gene expression evaluation of the $\alpha$-smooth muscle actin ( $\alpha$ SMA), smooth muscle 22- $\alpha$ (SM-22a), Calponin-1, and Caldesmon1 as SMC differentiation gene markers in SMC-differentiated human iPSCs while cultured on tissue culture polystyrene (TCPS), TCPS/curcumin,
SM22-a

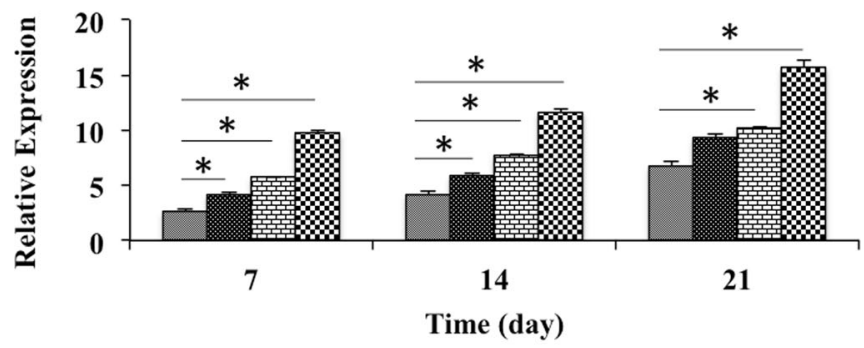

$\square$ TCPS 圆TCPS/curcumin $⿴ 囗 十$ Nanofibers $\mathbb{N}_{\text {Nanofibers/curcumin }}$

\section{Caldesmon1}

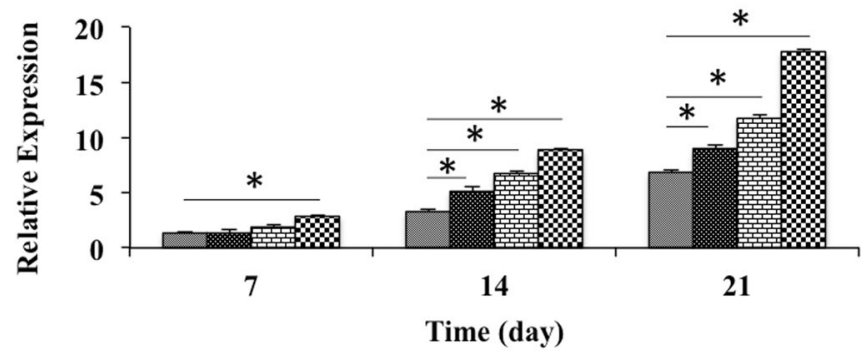

$\square$ TCPS TCPS/curcumin $\mathbf{⿴ 囗 十}_{\text {Nanofibers }}$ Nanofibers/curcumin chitosan/Col/PVA (nanofibers), and chitosan/Col/PVA/curcumin (nanofibers/curcumin) groups on days 7,14 , and 21 . The significant differences between groups are indicated with one star sign $(P<0.05)$. 
Figure 6. The $\alpha$-smooth muscle actin $(\alpha \mathrm{SMA})$ protein staining by ICC in SMC-differentiated human iPSCs while cultured on chitosan/Col/PVA (nanofibers) $(B)$ and chitosan/Col/PVA/ curcumin $(D)$ and DAPI staining was also performed for nanofiber $(A)$ and nanofiber/curcumin $(C)$ groups; ICC result quantification showed significant differences in expression of $\alpha \mathrm{SMA}$ protein between two groups $(E)$.
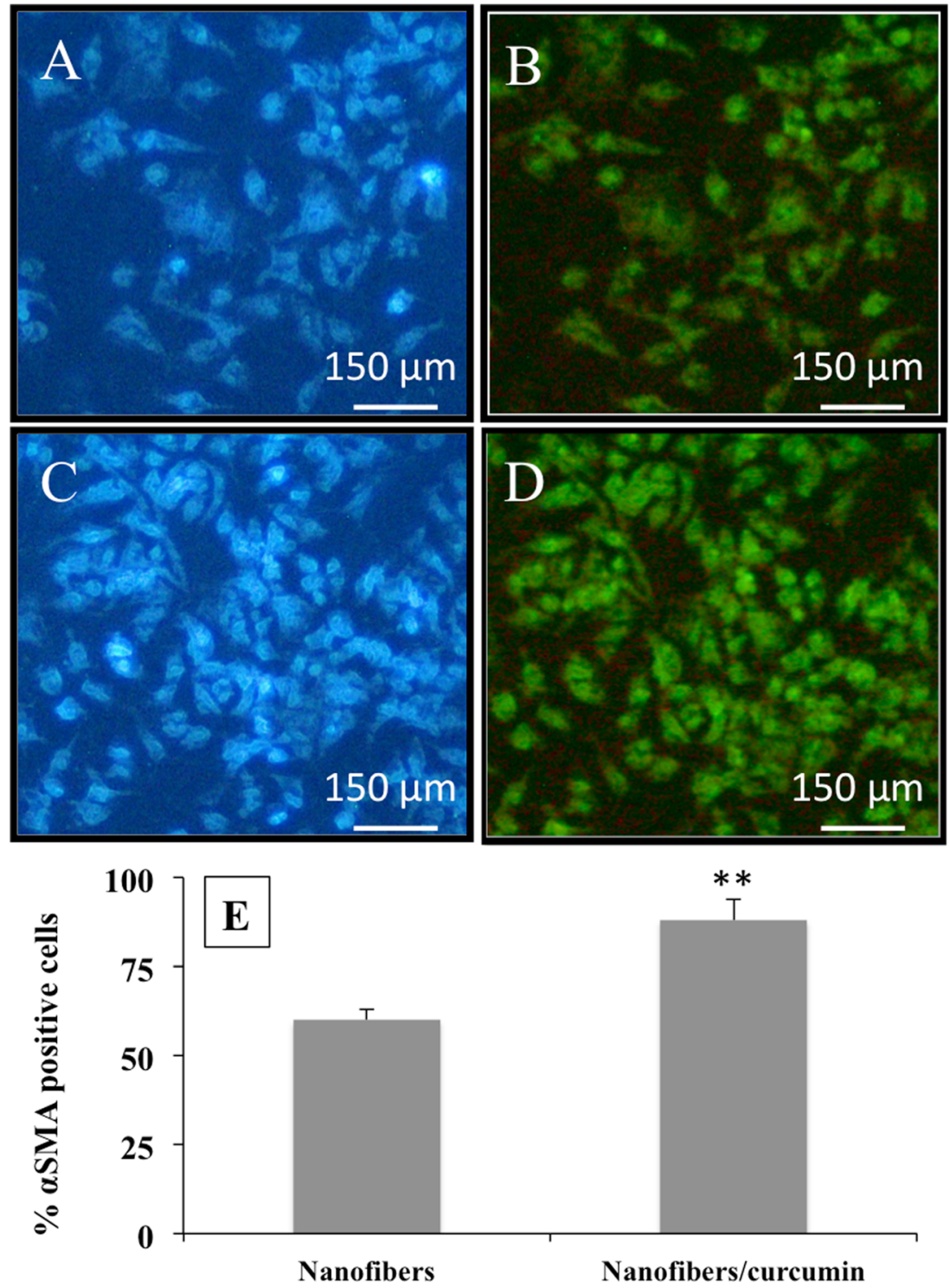

with the intended tissue transplant, but the results are usually not fully satisfactory due to immune suppression complications, increased number of failed transplants, and reduced number of organ donors (Abouna 2003; Greenwald et al. 2012). Therefore, discovering other ways to replace or repair damaged tissues and organs is essential. Regenerative medicine and tissue engineering are working to understand the mechanisms of tissue regeneration, and to find a way to regenerate damaged tissues (Wobma and Vunjak-Novakovic 2016). It can be possible to repair the function of vital organs by improving the ability of the tissue to regenerate itself, or by developing alternative biological tissues that are able to compensate for the correct function of the missing organs (Baddour et al. 2012). Our goal in this project was to design a nanofibrous scaffold with appropriate morphological, mechanical, and physiological properties for use in the bladder tissue engineering. Previously, we used Polyvinylidene fluoride (PVDF) (Ardeshirylajimi et al. 2018), poly lactic-co-glycolic acid (PLGA) (Mirzaei et al. 2019), and polyacrylonitrile/polyethylene oxide (PAN/PEO) (Fakhrieh et al. 2019) nanofibrous scaffolds for bladder tissue engineering, despite good results in stem cell differentiation into smooth muscle cells, but scaffolds were not mechanically appropriate for the bladder tissue engineering. Herein, we used a combination of natural and synthetic polymers to make the scaffold more similar to the bladder, chitosan was selected as a natural polymer with unique properties, collagen was selected as the other natural polymer found in abundance in the bladder matrix, and PVA as a synthetic polymer was also selected to improve structural and mechanical properties of the tissue engineering scaffold. In addition, a natural active ingredient called curcumin was also used to take advantage of its intrinsic properties to improve the stem cell differentiation process into bladder SMCs. Fabricated scaffolds demonstrated the fibrous structure with interconnected pores without any bead, with proper 
curcumin release and absolute biocompatibility for use in tissue engineering applications. In the presence of the curcumin, protein adsorption, cell attachment, and cell viability were increased significantly. However, the highest amounts of these parameters were detected in the curcumin-incorporated nanofiber group, which showed a synergic relationship between curcumin and nanofibrous structure. In agreeing with our results, Golchin et al. demonstrated that incorporating the curcumin in chitosan/PVA/ carbopol/PCL improved biological behavior of the nanofibers and in addition mesenchymal stem cell viability was also increased while cultured on the curcumin-incorporated nanofibers compared with those nanofibers without curcumin (Golchin et al. 2019).

The $\alpha$-SMA gene and protein expression are very important to the motility and contraction of the bladder matrix (Cherng et al. 2008) and our gene expression and ICC results revealed that the highest $\alpha$-SMA expression was detected in the human iPSCs cultured on the nanofiber/curcumin group compared with the nanofiber, TCPS/curcumin, and TCPS groups. In agreeing with us, Song et al. reported that $\alpha$ SMA gene and protein expression were increased significantly during SMC differentiation process of the dental pulp stem cells (Song et al. 2016).

SM22-a is one of the most primary SMC gene markers that its increased expression leads to regulating other SMC-related gene markers such as Calponin1. Our results indicated that the increase of SM22-a gene expression in iPSCs cultured on nanofiber/curcumin group was significantly higher than those cells cultured on the nanofiber, TCPS/curcumin, and TCPS groups. This expression pattern was also detected in Calponin1 and Caldesmonl genes, where these two genes were also expressed in differentiated cells in nanofiber/ curcumin group significantly higher than the cells differentiated on the nanofiber, TCPS/curcumin, and TCPS groups. In agreeing with our results, the increase in $\alpha$-SMA, SM22-a, Caldesmon1, and Calponin1 expression was reported by Jack et al. while they cultured adipose-derived stem cells on the surface of the PLGA electropulled microfibers (Jack et al. 2009).

\section{Conclusion}

According to the results obtained in this study, human iPSCs viability and its SMC differentiation potential were increased in the presence of curcumin. On the other hand, chitosan/Col/ PVA nanofibrous scaffold has also a great potential to support SMC differentiation of the human iPSCs. With the combination of these two factors by incorporating of curcumin in the nanofibers, SMC differentiation potential of the human iPSCs was significantly increased compared with the other groups. These characteristics of the fabricated nanofibers could also be the results of the presence of different natural and synthetic biopolymers in its structure.

Funding information This work was supported by the Shahid Beheshti University of Medical Sciences (Grant No. 17801).

\section{Compliance with ethical standards}

Conflict of interest The authors declare that they have no conflict of interest.

\section{References}

Abouna GM (2003) Ethical issues in organ and tissue transplantation. Exp Clin Transplant 1:125-138

Anand P, Kunnumakkara AB, Newman RA, Aggarwal BB (2007) Bioavailability of curcumin: problems and promises. Mol Pharm 4:807-818

Appasani K, Appasani RK (2011) Introduction to stem cells and regenerative medicine. Stem Cells Regen Med. Springer, pp 3-18

Ardeshirylajimi A, Ghaderian SM-H, Omrani MD, Moradi SL (2018) Biomimetic scaffold containing PVDF nanofibers with sustained TGF- $\beta$ release in combination with AT-MSCs for bladder tissue engineering. Gene 676:195-201

Atala A (2007) Engineering tissues, organs and cells. J Tissue Eng Regen Med 1:83-96

Atala A (2008) Tissue engineering of the urethra. Urethral Reconstr Surg. Springer, pp 337-345

Baddour JA, Sousounis K, Tsonis PA (2012) Organ repair and regeneration: an overview. Birth Defects Res C Embryo Today 96:1-29

Bengmark S (2006) Curcumin, an atoxic antioxidant and natural NfkB, cyclooxygenase-2, lipooxygenase, and inducible nitric oxide synthase inhibitor: a shield against acute and chronic diseases. J Parenter Enter Nutr 30:45-51

Caplan AI (2007) Adult mesenchymal stem cells for tissue engineering versus regenerative medicine. J Cell Physiol 213:341-347

Cherng S, Young J, Ma H (2008) Alpha-smooth muscle actin ( $\alpha$-SMA). J Am Sci 4:7-9

Dong C, Lv Y (2016) Application of collagen scaffold in tissue engineering: recent advances and new perspectives. Polymers 8:42

Fakhrieh M, Darvish M, Ardeshirylajimi A, Taheri M, Omrani MD (2019) Improved bladder smooth muscle cell differentiation of the mesenchymal stem cells when grown on electrospun polyacrylonitrile/polyethylene oxide nanofibrous scaffold. J Cell Biochem

Golchin A, Hosseinzadeh S, Staji M, Soleimani M, Ardeshirylajimi A, Khojasteh A (2019) Biological behavior of the curcumin incorporated chitosan/poly (vinyl alcohol) nanofibers for biomedical applications. J Cell Biochem

Green JJ, Elisseeff JH (2016) Mimicking biological functionality with polymers for biomedical applications. Nature 540:386

Greenwald MA, Kuehnert MJ, Fishman JA (2012) Infectious disease transmission during organ and tissue transplantation. Emerg Infect Dis 18:e1

Horst M, Eberli D, Gobet RM, Salemi S (2019) Tissue engineering in pediatric bladder reconstruction-the road to success. Front Pediatr 7 : 91

Islam S, Bhuiyan MAR, Islam MN (2017) Chitin and chitosan: structure, properties and applications in biomedical engineering. J Polym Environ 25:854-866 
Jack GS, Zhang R, Lee M, Xu Y, Wu BM, Rodríguez LV (2009) Urinary bladder smooth muscle engineered from adipose stem cells and a three dimensional synthetic composite. Biomaterials 30:3259-3270

Kadajji VG, Betageri GV (2011) Water soluble polymers for pharmaceutical applications. Polymers 3:1972-2009

Ma PX (2008) Biomimetic materials for tissue engineering. Adv Drug Deliv Rev 60:184-198

Marin E, Rojas J, Ciro Y (2014) A review of polyvinyl alcohol derivatives: promising materials for pharmaceutical and biomedical applications. Afr J Pharm Pharmacol 8:674-684

Mirzaei A, Saburi E, Islami M, Ardeshirylajimi A, Omrani MD, Taheri M, Moghadam AS, Ghafouri-Fard S (2019) Bladder smooth muscle cell differentiation of the human induced pluripotent stem cells on electrospun Poly (lactide-co-glycolide) nanofibrous structure. Gene 694:26-32

O’Brien FJ (2011) Biomaterials \& scaffolds for tissue engineering. Mater Today 14:88-95

Orabi H, Bouhout S, Morissette A, Rousseau A, Chabaud S, Bolduc S (2013) Tissue engineering of urinary bladder and urethra: advances from bench to patients. Sci World J 2013

Saburi E, Atabati H, Kabiri L, Behdari A, Azizi M, Ardeshirylajimi A, Enderami SE, Ghaderian SMH, Nafar M, Parvin M (2019a) Bone morphogenetic protein-7 incorporated polycaprolactone scaffold has a great potential to improve survival and proliferation rate of the human embryonic kidney cells. J Cell Biochem 120:9859-9868

Saburi E, Islami M, Hosseinzadeh S, Moghadam AS, Mansour RN, Azadian E, Joneidi Z, Nikpoor AR, Ghadiani MH, Khodaii Z (2019b) In vitro osteogenic differentiation potential of the human induced pluripotent stem cells augments when grown on Graphene oxide-modified nanofibers. Gene 696:72-79

Selim M, Bullock AJ, Blackwood KA, Chapple CR, MacNeil S (2011) Developing biodegradable scaffolds for tissue engineering of the urethra. BJU Int 107:296-302

Song B, Jiang W, Alraies A, Liu Q, Gudla V, Oni J, Wei X, Sloan A, Ni L, Agarwal M (2016) Bladder smooth muscle cells differentiation from dental pulp stem cells: future potential for bladder tissue engineering. Stem Cells Int 2016

Takahashi K, Yamanaka S (2006) Induction of pluripotent stem cells from mouse embryonic and adult fibroblast cultures by defined factors. Cell 126:663-676

Wobma H, Vunjak-Novakovic G (2016) Tissue engineering and regenerative medicine 2015: a year in review. Tissue Eng B Rev 22:101113 